\title{
Cervical Spine and Degenerative Conditions
}

\author{
Afsoun Seddighi, Amir Hasan Hoseini, Ashkan Divanbeygi, Sajjad Alizadeh* \\ Functional Neurosurgery Research Center, Shohada Tajrish Comprehensive Neurosurgical Center of Excellence, Shahid \\ Beheshti University of Medical Sciences, Tehran, Iran
}

\begin{abstract}
Degenerative disks and cervical spine ligaments diseases caused by the collapse of the anatomical position of vertebral structures and attempt to adapt. The Degenerative disorder of the neck disks has a prevalence of $20 \%$ to $25 \%$ in the population under the age of 59 and $70 \%-95 \%$ in the population 65 years and older. This study performed as a retrospective by reviewing the clinical files of patients with anterior neck discectomy in Shohada Tajrish hospital between 2014-2017. The severity of pain in the type of radiculopathy and neck pain measured from 1 to 10 based on the visual analog scale (VAS). The most common clinical manifestations of degenerative and cervical disks are neck pain, radiculopathy, and cervical myelopathy, alone or in combination. Generally, the disc hernia suddenly associated with acute symptoms.

Keywords: Cervical disk; Degenerative disorder; Pain.
\end{abstract}
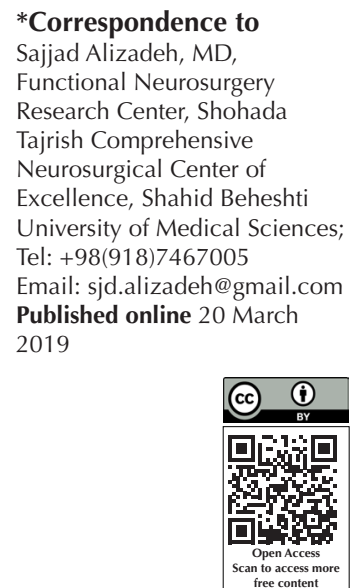

Citation: Seddighi A, Hoseini AH, Divanbeygi A, Alizadeh S. Cervical spine and degenerative conditions. Int Clin Neurosci J. 2019;6(1):1-5. doi: 10.15171/icnj.2019.01.

\section{Introduction}

Degenerative disks and cervical spine ligaments diseases caused by the collapse of the anatomical position of vertebral structures and attempt to adapt. This process leads to structural changes in the joints involved. Calcification and thickening of the ligament occur, and small abnormal bones appear in the form of small appendages. ${ }^{1,2}$ Cervical spondylitis is the most commonly used term to describe degenerative disks of the neck. Its primary definition is the formation of secondary osteophyte to degenerative disks of the neck. These diseases are more prevalent in adults and are often related to age. The Degenerative disorder of the neck disks has a prevalence of $20 \%$ to $25 \%$ in the population under the age of 59 and $70-95 \%$ in the population 65 years and older. However, only a few patients are symptomatic. Radiographic manifestations in favor of spondylitis found in $25 \%-50 \%$ of the population under 50 and $75 \%-85 \%$ of the population over 65 years of age. The incidence of cervical spondylitis in the graph of asymptomatic patients was $95 \%$ in men and $70 \%$ in women in the seventh year of life. The onset of symptoms is usually from the 3 rd to the 6th decade, and men are more likely than women, and most respond to medical and non-surgical treatments during transient pain. A discectomy performed when the severity of the symptoms is unbearable, or if there is no response to non-surgical treatments or a significant risk of injury to the spinal cord. ${ }^{1,3}$ The purpose of this study was to evaluate the distribution of complaints and the severity of neurological symptoms in patients before surgery.

\section{Materials and Methods}

This study performed as a retrospective by reviewing the clinical files of patients with anterior neck discectomy in Shohada Tajrish hospital between 2014 and 2017. The duration of the onset of the symptoms until the start of the first symptom of the patients before surgery was based on the day and converted to the month. Signs and symptoms of the patients identified in four ways: radiculopathy, myelopathy, radiculomyelopathy, and cervical pain. The severity of pain in the type of radiculopathy and neck pain measured from 1 to 10 based on the visual analog scale (VAS). The preoperative therapeutic measures extracted from the questionnaires. Cervical spine curvature determined by Cobb angle. In this way, if this angle were more significant than +5 degrees, the Kyphosis would be, and if there was less than five, the cervical Lordosis was present. We considered these 2 numbers equal to the straight line state. ${ }^{4}$ Descriptive statistics in SPSS version 20 software analyzed data.

\section{Results}

One hundred ninety-eight had entry criteria. The number of men and women was 118 and 80 , respectively. The mean age of the patients was $50.48 \pm 13.12$ in the range of $25-70$ years. The average duration of the first symptom before 
surgery was $3.20 \pm 1.52$ months. 86 (43.4\%) were smokers. In $100 \%$ of cases, the first symptom was cervical pain. 124 (62.16\%), 72 (36.24\%) and $12(6.11 \%)$ had radiculopathy, radiculomyelopathy, and myelopathy respectively. 108 (54.54\%) intervertebral in one level, 90 (45.45\%) had two levels. Most intervertebral spaces were involved with C6C7 and C5-C6. The level of intervertebral spatial conflict shown in Figure 1.

The severity of cervical pain was $7.81 \pm 1.88$, and $7.17 \pm 1.36$ for radicular pain severity by VAS. 15 patients (14.6\%) and 47 cases (45.6\%) had cervical keratitis and lordosis, respectively. The mean of neck lordosis was $4.22 \pm 2.88$ degree.

The frequency of medical procedures performed for patients before surgery presented in Figure 2.

There was a significant reverse linear relationship between radicular pain intensity and lordosis $(P<0.05)$. There was no significant correlation between cervical pain intensity, smoking, and lordosis.

\section{Discussion and Conclusion}

The most common clinical manifestations of degenerative and cervical disks are neck pain, radiculopathy, and cervical myelopathy, alone or in combination. ${ }^{5,6}$ Generally, the disc hernia suddenly associated with acute symptoms. Symptoms have often seen in spondylosis.?

\section{Cervical Pain}

Cervical pain is common in the back region and focuses on the para spinous muscles. Patients usually suffer from pain in the area or between the 2 scapulae and the occipital headache. Often, the pain intensifies with movement. Cervical pains are often causing by diseases involving the fascia joints, disks, dilatation and dislocation of ligaments, and segregation instability or a combination of all of these. The sinuvertebral nerve located at the base of the anus in the posterior, around and posterior longitudinal ligament. This nerve connects ventral ramus and gray ramus. There are branches of gray ramus in the anterior region of the disk and longitudinal ligament. The stimulation of each of these buildings leads to neck pain. Contrary to the appearance that degenerative diseases should always accompany by cervical pain, it has reported that in $4.5 \%$ to $18.2 \%$ of cases, the patients may be asymptomatic and have no cervical pain. The reason for this issue is still unclear. ${ }^{2,5,8,9}$

\section{Cervical Radiculopathy}

Hernia discs or osteophyte changes in spondylosis can cause pain in the nervous system or radiculopathy. Despite a variety of stressors in the nerves, symptoms are common. Significant radicular pain is the presence of proliferative cervical and arthroplasty pains in the nervous system. ${ }^{1}$

Typically, acute radicular pains see in a sudden hernia of the disc or the trauma. Radiculopathy in spondylosis is

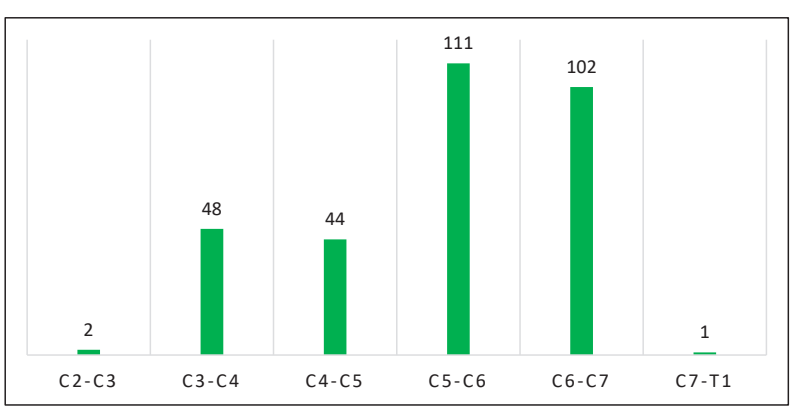

Figure 1. The Level of Intervertebral Spatial Conflict.

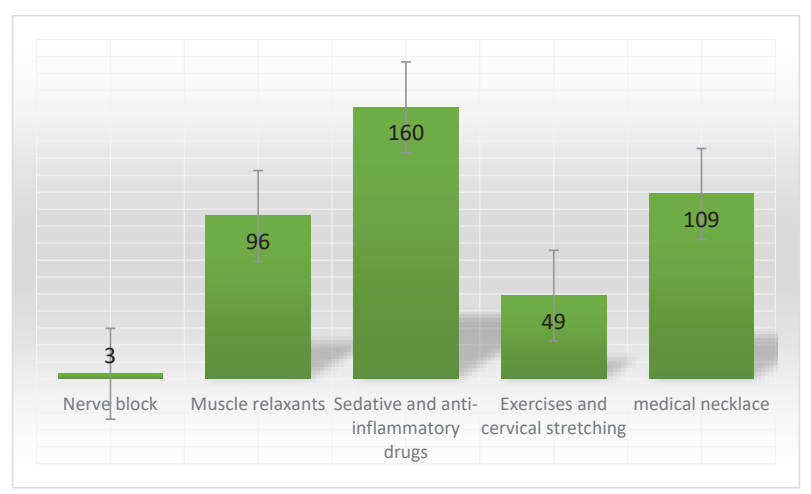

Figure 2. Frequency of Non-surgical Treatment Proceedings.

more common in chronic displays or periodic attacks. In the area covered by the affected nerve, there is usually a sensory disorder in the touch and feel of the abscess. Most of the sensory and motor disorders have seen together, but each one may also occur alone. Fasciculation and weakness have commonly seen in chronic cases. Given that it usually involves more than one segment in the spondylosis, the symptoms are usually more severe and extensive than that found in a single disc hernia. ${ }^{1,2}$

The intercostal space C5-C6 and C6-C7 are the most commonly involved levels. In the cervical spine, the roots of the nerves go out above their same spines. For example, in the herniation of the $\mathrm{C} 5-\mathrm{C} 6$, the root of the $\mathrm{C} 6$ nerve is pressurized and induces radiculopathy of the C6. In this radiculopathy, there is back pain in the neck that extends to the muscle of the armpit, the ankle-arm, the upper part of the forearm, and the back of the hand, thumb and pointing finger. Turning the head, bending the sides, and pressure on vortex (Spurling's sign) will exacerbate the shoulder and reduce the pain. The weakness of the elbow flexor muscles and the wrist exteriors is usually present in an accurate neurological examination, even before the patient becomes aware of the symptoms. Reduction or loss of biceps muscle reflex is strongly in favor of the diagnosis of C6 radiculopathy. Sensory changes are diverse and usually occur on the back of the hand on the thumb and middle fingers - the disc herniation or degeneration of the C6-C7 space results in radial C7 radiculopathy. There 
is a pain in the back of the shoulders and neck, and it spreads to the triceps muscle and the outer back of the forearm and middle finger. Spread of pain to the finger and the ring can also achieve. The refolding of the triceps of the arm is reduced or eliminated, and it is possible that the elbow extensors and wrist flexors and finger extensors in the metacarpophalangeal joint have weakened. Various sensory changes are also present here, often seen in the C7 dermatome, which includes the middle region of the middle hands and middle finger. C8 radiculopathy is characterized by the diffusion of pain along the middle of the forearm and by propagation towards the small finger and the ring. There is usually a sensory disorder in the same area, and the lack of intricate muscles is evident. C5 radiculopathy had a small release and associated with fatal pain from the neck to the shoulder. Deltoid muscle weakness and sensory changes in the deltoid region may be present. $\mathrm{C} 3$ and $\mathrm{C} 4$ radiculopathies are not common and often associated with pain and anesthesia in the mastoid region (C3) and the back of the neck (related to $\mathrm{C} 4)$. There is no significant muscle weakness in these two cases. ${ }^{1,2,6,10}$

Pain patterns, sensory changes, and muscle weakness are critical factors in reaching the diagnosis. It should note that in some cases, such as overlapping of nerve roots, anatomical variations, and chronic and multi-level diseases, it is difficult to make a diagnosis. The annual incidence of cervical radiculopathy is 83.2 per 100000 people. The incidence of radiculopathy in degenerative diseases of the cervical disc is $22 \%-68 \%$. $^{5,6,9}$

Usually, the curvature of the cervical spine is lordosis. In the dorsal region of the cervical vertebrae, a straight line has drawn between the $\mathrm{C} 2$ and $\mathrm{C} 7$ in the dorsocaudal regions. When no parts of the $\mathrm{C} 3$ and $\mathrm{C} 7$ vertebral bodies stop this line, the cervical spine is in the lordosis. When a part of the $\mathrm{C} 3$ and $\mathrm{C} 7$ vertebral bodies cut off the line between $\mathrm{C} 2$ and $\mathrm{C} 7$, the cervical spine is in kyphosis. The presence of kyphosis in the cervical spine is abnormal and can cause pressure or exacerbation of the pressure on the nerve building and cause radicular pains and myelopathy. ${ }^{11,12}$

\section{Cervical Myelopathy}

Spondylosis and degenerative disc disease can cause the spinal syndrome. Complete spinal cord injury characterized by the loss of all sensory and motor levels of the lesion site. This injury is not common, but it can occur in a large disc herniation or the course of a mild trauma in the presence of spondylosis and canal stenosis. ${ }^{7}$

The incomplete spinal cord injury and its syndromes have more commonly seen than the full type. These syndromes include central spinal cord syndrome, BrownSequard syndrome and anterior spinal cord syndrome. These syndromes can create in isolation or conjunction with an acute injury. The central spinal cord syndrome characterized by severe loss of muscular strength of the upper extremity relative to the lower extremity and varying degrees of sensory disturbances. Classically, this disordered pattern is related to that part of the spinal cord, which has arm and hand threads in the corticospinal tracts. They diagnosed Brown's syndrome with hemiparesis on the side of the lesion, the loss of the sense of position and vibration of the same side, and the loss of the sense of heat and side pain. Anterior spinal cord syndrome characterized by immediate and complete cervical spinal cord injury following acute spinal cord injuries and relative loss of touch and vibration. The critical point of this syndrome is that it usually improves with the actions of decompressive and the removal of pressure resulted in symptoms of the patients. ${ }^{7,9,13}$

The cervical spondylitis myelopathy is referred to as spinal cord stenosis due to degenerative changes in the cervical spine. This is the most severe and vital complication of degenerative disc disease and neck ligament and leads to spinal cord dysfunction, especially in patients over the age of 55 years. The cervical myelopathy, in the beginning, shows up with the disturbance in walking and weakness of the hands. Lower extremity spasms play an essential role in walking. Disruption of walking is usually mysterious and has a slow progression. When spasticity and muscle weakness increase, walking problems are evident. The progress of this process leads to severe consequences so that the person ultimately needs a wheelchair. Hand muscle weakness and sensory impairment result in loss of excellent motor skills. In practice, everyday life activities, such as closing the button of shirt or writing, are often disrupted. Neurological examination of these patients characterized by weakness of the upper extremities and hands, proximal weakness of the lower extremity, hyperreflexia, and regional disorientation. In $25 \%$ of cases of cervical myelopathy, patients with neck flexion experience a similar sensation of electrocution and paresthesia known as Lhermitte's sign. Bladder, intestinal and dysfunctional disorders in sexual activity have also seen in severe cervical myelopathy. The incidence of these disorders reported in $5 \%-10 \%$ of studies. ${ }^{1,7,14}$

\section{Medical and Non-surgical Treatments}

Although patients with advanced neurodevelopmental defects of the compression of the nerves and spinal cord routinely require surgical intervention, non-surgical and medical methods have also used in the treatment of patients with cervical and arm pain. In cases where there is no immediate indication of surgery, medical and nonsurgical treatments should first use. The basis of medical and non-surgical treatments should be the reduction of stress on the nerves and spinal cord., ${ }^{2,9}$

The majority of patients with cervical, articular, or both non-surgical and medical treatments are adequately controlled and ultimately improved. Nonsurgical treatments include special exercises, cold and heat therapy, stretching and rehabilitation exercises, 
drug therapy, epidural injections, ozone therapy, laser therapy and neurological stimulation from the skin. Cervical radiculopathy often responds to resting-control treatments, non-steroidal anti-inflammatory drugs, exercise therapy, and rehabilitation over time. However, cervical myelopathy usually develops despite control therapies. ${ }^{5,9,14,15}$

Using a soft medical necklace for one week reduces pain and neck spasm. It should note that this type of neck does not entirely restrict the movement of the neck. This recovery can be achieved by stimulating mechanisms or by inducing local heat resulted in the medical necklace on the skin. Treatments based on immobility should not continue until the patients are brace assisted. In this type of treatment, daily isometric exercises in the neck significantly reduce tone and muscle atrophy. These exercises include stretching exercises for the shoulder and neck muscles against constant resistance without cervical spine attachment and movement. Stretching treatments that have a long history in patients with mild to moderate cervical spondylosis reduce pain in $81 \%$ of cases. Aerobic exercises with flexibility and advanced endurance exercise for shoulder, upper and neck belts are used to strengthen and support the cervical spine. ${ }^{16,17}$ Creating changes in everyday life habits such as weight loss, smoking cessation, stress and anxiety control, occupational status and ergonomic evaluation play an essential role in the long-term management of patients. ${ }^{14,18}$

Transcutaneous electrical nerve stimulation is also useful in reducing chronic neck pain and radiculopathy. The mechanism of its exact effect is unknown, but it said that this stimulation activates the areas of analgesia in the brain stem. Activates the spinal cord gating mechanism and prevents the peripheral nerve fibers involved in transmitting pain. Correct insertion of electrodes in this method is significant. The duration of treatment is usually 2 weeks, during which time it determined whether the patient responds or does not. The side effects of this method are local skin irritation. ${ }^{19}$

The nervous block of the steroid and anesthetics injections done into the epidermis from the posterior part and through the ligament flaming. This type of treatment is useful in acute attacks, but long-term use of corticosteroids has not usually recommended. Injection of ozone into intervertebral space has also reported for the treatment of degenerative disc disease in acute type. ${ }^{20,21}$

Ant arrhythmias with broad spectrum, a non-steroidal anti-inflammatory, muscle relaxants, and corticosteroids can mention in the treatment of acne or chronic degenerative disc disease. ${ }^{9}$

The results of this study, while explaining the clinical status of patients before neck discectomy surgery, brings up for faster referral of patients to surgery.

\section{Conflict of Interest Disclosures}

The authors declare that they have no conflict of interests.

\section{Ethical Statement}

Not applicable.

\section{References}

1. Harrop JS, Hanna A, Silva MT, Sharan A. Neurological manifestations of cervical spondylosis: an overview of signs, symptoms, and pathophysiology. Neurosurgery. 2007;60(1 Suppl 1):S14-20. doi: 10.1227/01.neu.0000215380.71097. ec.

2. Wong JJ, Cote P, Quesnele JJ, Stern PJ, Mior SA. The course and prognostic factors of symptomatic cervical disc herniation with radiculopathy: a systematic review of the literature. Spine J. 2014;14(8):1781-9. doi: 10.1016/j.spinee.2014.02.032.

3. Kato F, Yukawa Y, Suda K, Yamagata M, Ueta T. Normal morphology, age-related changes and abnormal findings of the cervical spine. Part II: Magnetic resonance imaging of over 1,200 asymptomatic subjects. Eur Spine J. 2012;21(8):1499507. doi: 10.1007/s00586-012-2176-4.

4. Rohani Y, Divanbeigi A, Taheri T, Elahi F. Prospective Comparative Study of Anterior Cervical Discectomy Alone, With Iliac Crest Bone Graft Fusion and PEEK Cage Fusion. Daneshvar Medicine. 2009;16(80):35-42.

5. Cohen SP. Epidemiology, diagnosis, and treatment of neck pain. Mayo Clin Proc. 2015;90(2):284-99. doi: 10.1016/j. mayocp.2014.09.008.

6. Kim KT, Kim YB. Cervical radiculopathy due to cervical degenerative diseases: anatomy, diagnosis and treatment. J Korean Neurosurg Soc. 2010;48(6):473-9. doi: 10.3340/ jkns.2010.48.6.473.

7. Lebl DR, Hughes A, Cammisa FP Jr, O'Leary PF. Cervical spondylotic myelopathy: pathophysiology, clinical presentation, and treatment. Hss j. 2011;7(2):170-8. doi: 10.1007/s11420-011-9208-1.

8. Morishita $\mathrm{Y}$, Naito $\mathrm{M}$, Hymanson $\mathrm{H}$, Miyazaki $\mathrm{M}, \mathrm{Wu} \mathrm{G}$, Wang JC. The relationship between the cervical spinal canal diameter and the pathological changes in the cervical spine. Eur Spine J. 2009;18(6):877-83. doi: 10.1007/s00586-0090968-y.

9. Todd AG. Cervical spine: degenerative conditions. Curr Rev Musculoskelet Med. 2011;4(4):168-74. doi: 10.1007/s12178011-9099-2.

10. Wilson JR, Barry S, Fischer DJ, Skelly AC, Arnold PM, Riew KD, et al. Frequency, timing, and predictors of neurological dysfunction in the nonmyelopathic patient with cervical spinal cord compression, canal stenosis, and/ or ossification of the posterior longitudinal ligament. Spine (Phila Pa 1976). 2013;38(22 Suppl 1):S37-54. doi: 10.1097/ BRS.0b013e3182a7f2e7.

11. Grob D, Frauenfelder H, Mannion AF. The association between cervical spine curvature and neck pain. Eur Spine J. 2007;16(5):669-78. doi: 10.1007/s00586-006-0254-1.

12. Stulik J, Nesnidal P, Sebesta P, Vyskocil T, Kryl J. [Kyphotic deformities of the cervical spin]. Acta Chir Orthop Traumatol Cech. 2011;78(3):215-24

13. Nagata K, Yoshimura N, Hashizume $\mathrm{H}$, Muraki S, Ishimoto $\mathrm{Y}$, Yamada $\mathrm{H}$, et al. The prevalence of cervical myelopathy among subjects with narrow cervical spinal canal in a population-based magnetic resonance imaging study: the Wakayama Spine Study. Spine J. 2014;14(12):2811-7. doi: 10.1016/j.spinee.2014.03.051.

14. Rhee JM, Shamji MF, Erwin WM, Bransford RJ, Yoon ST, Smith JS, et al. Nonoperative management of cervical myelopathy: a systematic review. Spine (Phila Pa 1976). 2013;38(22 Suppl 1):S55-67. doi: 10.1097/BRS.0b013e3182a7f41d.

15. Avery RM. Massage therapy for cervical degenerative disc disease: alleviating a pain in the neck? Int J Ther Massage 
Bodywork. 2012;5(3):41-6.

16. Gross A, Kay TM, Paquin JP, Blanchette S, Lalonde P, Christie T, et al. Exercises for mechanical neck disorders. Cochrane Database Syst Rev. 2015;1:Cd004250. doi: 10.1002/14651858.CD004250.pub5.

17. Kuijper B, Tans JT, Beelen A, Nollet F, de Visser M. Cervical collar or physiotherapy versus wait and see policy for recent onset cervical radiculopathy: randomised trial. BMJ. 2009;339:b3883. doi: 10.1136/bmj.b3883.

18. Gross AR, Kaplan F, Huang S, Khan M, Santaguida PL, Carlesso LC, et al. Psychological Care, Patient Education, Orthotics, Ergonomics and Prevention Strategies for Neck Pain: An Systematic Overview Update as Part of the ICON Project. Open Orthop J. 2013;7:530-61. doi: 10.2174/1874325001307010530.

19. Kroeling P, Gross A, Graham N, Burnie SJ, Szeto G, Goldsmith $\mathrm{CH}$, et al. Electrotherapy for neck pain. Cochrane Database Syst Rev. 2013(8):Cd004251. doi: 10.1002/14651858. CD004251.pub5.

20. Alexandre A, Coro L, Azuelos A, Buric J, Salgado H, Murga M, et al. Intradiscal injection of oxygen-ozone gas mixture for the treatment of cervical disc herniations. Acta Neurochir Suppl. 2005;92:79-82

21. Falco FJ, Datta S, Manchikanti L, Sehgal N, Geffert S, Singh V, et al. An updated review of the diagnostic utility of cervical facet joint injections. Pain Physician. 2012;15(6):E807-38. 